Gdańsk 2020, Nr. 42

https://doi.org/10.26881/sgg.2020.42.06

Ewelina Kamińska-Ossowska

(Uniwersytet Szczeciński / Universität Szczecin)

https://orcid.org/0000-0001-8028-9577

\title{
Polnische germanistische Forschung zur Kinder- und Jugendliteratur nach 1989
}

\begin{abstract}
Das Phänomen ,Kinder- und Jugendliteratur' wurde lange Zeit in der Forschung polnischer Germanistinnen und Germanisten relativ selten wahrgenommen. Im Beitrag wird der Versuch unternommen, die Frage nach den etwaigen Veränderungen in diesem Bereich zu beantworten und, unter Berücksichtigung von polnischen germanistischen Zeitschriften und Buchpublikationen, die polnische germanistische Forschung zur KJL nach 1989 zu charakterisieren.
\end{abstract}

Schlüsselwörter: Kinder- und Jugendliteratur, polnische Germanistik, Forschung und Lehre

Polish German scholars' research on children's and youth literature after 1989. The phenomenon of children's literature and young adult literature has relatively rarely been made the object of research of Polish Germanists. This essay attempts to show the changes in this field. Based on Polish specialist periodicals and publications in the field of German Studies, the aim of the paper is to examine and characterize the research of Polish Germanists after 1989.

Keywords: children's literature and young adult literature, Polish German Studies, research and teaching

In dem Beitrag wird der Versuch unternommen, den Stellenwert der deutschsprachigen Kinder- und Jugendliteratur (im Folgenden KJL abgekürzt) in der Forschung und Lehre polnischer Germanistinnen und Germanisten zu ermitteln. Es werden einleitend die mit den gesellschaftlichen Modernisierungsprozessen verbundenen Veränderungen der KJL kurz charakterisiert, dann werden Überlegungen zum Spezifikum der Auslandsgermanistik und deren Literaturseminare angestellt, schließlich wird ein Überblick über die Präsenz der KJL in polnischer germanistischer Forschung und Lehre gegeben.

\section{Zur Geschichte und Gegenwart der KJL}

In der Aufklärung wurden Kinder und Jugendliche als eine separate Gruppe der Gesellschaft wahrgenommen, die auch eine altersspezifische sowie Erziehung und Bildung fördernde Literatur braucht. Seit dieser Zeit datiert die Assoziation, die KJL diene primär 
didaktischen Zwecken (Erziehung zur Literatur und Erziehung durch Literatur) und weise oft niedrigeren literarischen und ästhetischen Anspruch auf, was ihr deshalb eher abwertende Konnotationen mit Unterhaltungs- bzw. Trivialliteratur bringt. Eine Veränderung dieser Sichtweise beginnt infolge der gesellschaftlichen Modernisierungsprozesse, insbesondere der Umwälzungen der 1960er-1970er Jahre in den westeuropäischen Ländern. Sie finden in der „egalitaristischen, d. h. Kinder und Erwachsene annähernden, gleichstellenden Grundhaltung" (Ewers 1995: 21) in der Literatur, u. a. in Bezug auf die Autoritätsverhältnisse und Offenheit in der Darstellung des bisher Tabuisierten, ihren Niederschlag. ${ }^{1}$ Unter den Buchfiguren erscheinen nun anstelle der früheren kleinen Lieblinge: Rotznasen (vgl. den Titel des Beitrags von Hohmeister 1995), Individualisten, Aufsässige und Außenseiter. Die veränderte Thematik (,Was') verlangt nach neuen Formen der Darstellung (,Wie'). „Auf eine dezidiert kindgemäße folgt eine Kinderliteratur, die sich in formaler und stilistischer Hinsicht > emanzipiert<, d. h. auf die Seite der Erwachsenenliteratur der gesellschaftlichen Moderne gestellt hat." (Ewers 1995: 22) Unter den Darstellungstechniken findet man nun die moderne Ich-Erzählung, das personale Erzählen, den inneren Monolog oder Bewusstseinsstrom, realistische Erzählformen (Ewers 1995: 23-24), auch Rückblenden und Vorausdeutungen anstelle einer linearen Handlung, die den Wechsel der Zeitebenen und Erzählformen voraussetzen, sowie Collage und Montage (Gansel 1995: 13). In den 1980er Jahren kommt es zu einer „Modifikation des kinderliterarischen Realismus [...]. Neben den weiterhin präsenten sozialen Realismus tritt der psychologische Realismus“" (Stanz/Weinmann 2002: 127), die Thematik kreist um Ich-Stabilisierung und Selbstfindung, es setzt sich der „psychologische Kinderroman“ durch. Eine weitere Entwicklung der KJL ist - ebenfalls im Zuge der gesellschaftlichen Modernisierungsprozesse - in der zweiten Hälfte der 1990er Jahre zu beobachten. Die Lebensphase Jugend verlängert sich, die Übernahme der bisher üblichen Pflichten als Arbeitnehmer oder Elternteil erfolgt später - für die KJL bedeutet dies eine Erweiterung des Empfängerkreises, die traditionelle Jugendliteratur wird um die Literatur für junge Erwachsene (die Literaturwissenschaftler übernehmen diese Kategorie von den Soziologen, engl.young adults) ergänzt. In dieser Zeit erlebt die sog. Neue Pop-Literatur ihren Höhepunkt (Degler/Paulokat 2008). Zum besonderen Phänomen wird noch die All-Age- bzw. Crossover-Literatur, die die altersbedingte Bestimmung der Lesergruppen aufhebt und an mehrere Generationen zugleich adressiert sein kann; populär sind dual editions, d. h. Ausgaben, in denen derselbe Text in einem anderen Cover für Jugendliche und Erwachsene herausgegeben wird.

Die in den jeweiligen Jahrzehnten stattfindenden Veränderungen der KJL, der medialen Umwelt und auch der Leserkreise trugen zur Neudefinierung von tradierten und zur Herausbildung einer Reihe von neuen Gattungen bei. Die KJL

\footnotetext{
„In der neuen Kinderliteratur ab 1970 werden die Kinder aus ihren exotischen Spiel- und Abenteuerwelten ins wirkliche Leben zurückgeholt, [...]. Konsequenterweise macht sich die neue Kinderliteratur an die thematische Rückeroberung des Gesellschaftlichen bzw. des Politischen, [...]“ (Ewers 1995: 19). „Kleinbürgerliche Tabus verschwanden. Kindern und Jugendlichen wurde[n] in ihrer Literatur Themen zugestanden, mit denen sie in Wirklichkeit längst leben mußten. Kinder-und Jugendliteratur wurde als Teil der allgemeinen Literatur begriffen [...]." (Schönfeldt 1995: 125).
} 
hat mittlerweile sehr verschiedene Facetten [...]:

1. Die Gesamtheit der für Kinder und Jugendliche als geeignet empfundenen Literatur (intentionale $\mathrm{KJL})$.

2. Die Gesamtheit der für Kinder und Jugendliche geschriebenen fiktionalen und nichtfiktionalen Texte (spezifische KJL).

3. Die Gesamtheit der von Kindern und Jugendlichen rezipierten fiktionalen und nichtfiktionalen Texte (Kinder- und Jugendlektüre).

4. Ein Teilsystem des gesellschaftlichen Handlungs- bzw. Sozialsystems ,Literatur ‘ (,Subsystem KJL'). (Gansel 2016: 13).

Die Palette der Texte ist imponierend. Sie umfasst u. a. realistische KJL, phantastische KJL, Kinderlyrik, das Bilderbuch, Märchen, Mythen und Sagen, Fabeln, das Tierbuch, den psychologischen Kinderroman, den Adoleszenzroman, religiöse KJL, Abenteuerliteratur, geschichtliche KJL, zeitgeschichtliche KJL, Comics, Krimis, Sciencefiction. Die Liste können noch außerliterarische bzw. literaturverwandte an Kinder und Jugendliche adressierte Formen ergänzen: Theaterstücke, Kinderfunk, Kinderfilm, Kinderfernsehen, Zeitschriften und Sachbücher. ${ }^{2}$

\section{KJL in der germanistischen Forschung und Lehre in Polen}

Das Phänomen ,Kinder- und Jugendliteratur' ist in der Lehre und Forschung polnischer Germanistinnen und Germanisten präsent, doch lange Zeit war es lediglich eine Randerscheinung. Dieser Umstand resultierte u. a. aus der Situation der Germanistik in Polen nach 1945 - die Beschäftigung mit der deutschen Sprache, Literatur und Kultur wurde infolge der geschichtlichen Erfahrungen quasi zu einem politischen Thema. In den ersten Jahren gab es nur einzelne Universitäten, an denen der Bereich Germanistik, meistens als Lehrstuhl organisiert, angeboten wurde. Zudem wurden noch die meisten Lehrstühle bzw. Institute für Neuphilologie 1952 geschlossen und durften erst in der zweiten Hälfte der 1960er Jahre ihre didaktische Tätigkeit wiederaufnehmen. Es entstand eine Forschungslücke. In dieser Periode befassten sich jedoch mit der deutschsprachigen Literatur stellvertretend Polonisten, was die Thematik gewissermaßen vor dem Vergessen rettete. Typisch für eine Auslandsgermanistik war die Orientierung auf Sprachunterricht, fast überall wurde in erster Linie die Ausbildung von Deutschlehrern angestrebt, man bot aber obligatorische Kurse in (Geschichte der deutschen) Literatur und Sprache an. Der Literaturunterricht an Oberschulen war hauptsächlich polenzentriert, bei den Germanistikanwärtern konnte keine Kenntnis der deutschsprachigen Literatur vorausgesetzt werden, deshalb wurden für gewöhnlich Lehrveranstaltungen abgehalten, in denen ein Überblick über die literarischen Epochen, die wichtigsten Autoren und deren Werke gegeben wurde. Bis in die 1970er Jahre hinein warf man der KJL literarische Minderwertigkeit vor, wohl deshalb beobachtete man auch in der literaturwissenschaftlichen Wahrnehmung eine sog. Ghettoisierung - so die damalige Reflexion über die künstlich gezogene Grenze und die Distanz zwischen ihr und der Allgemeinliteratur (Doderer 1981: 9-17; 1992: 30-33). In der polnischen Germanistik war dies länger der Fall, die Studenten bekamen

\footnotetext{
2 Zu den Gattungen (Lange 2002a: 158-565), zu Medien und Sachbuch (Lange 2002b: 586-656).
} 
Informationen zu einem Kanon der Texte von herausgehobenem Wert, dagegen wurden die für junge Leser bestenfalls erwähnt, so dass sie in der akademischen Lehre fehlten. Der Durchschnittsstudent der polnischen Germanistik assoziierte mit der Kinderliteratur vor allem Märchen, kannte wohl den Namen Grimm aus der Privatlektüre und dem Unterricht, wahrscheinlich noch Karl May und Erich Kästner, deren Werke in polnischen Übersetzungen vorliegen, vielleicht auch Romane von Erwin Strittmatter (z. B. Tinko 1954, Pony Pedro 1959), Alfred Wellm oder Benno Pludra, die vor 1989 möglicherweise von den Dozenten aus der DDR erwähnt wurden. Seine Chancen, Einblick in die späteren, gezielt für Kinder und Jugendliche geschaffenen Texte, speziell in die im Westen entstehende problemorientierte, realistische Kinder- und Jugendliteratur (z. B. in die nach 1970 die Veränderungen der westeuropäischen Gesellschaften reflektierenden Romane von Peter Härtling, Ursula Wölfel, Renate Welsh, Christine Nöstlinger) zu bekommen, waren eher gering. Dieses EpochenKanon-Modell der Literaturseminare und -vorlesungen besteht zwar an polnischen Germanistikinstituten oft bis heute, allerdings wurden Ende der 1990er Jahre Fächer zur Wahl eingeführt und somit die Themenangebote erweitert: Die an der Kinder- und Jugendliteratur interessierten Lehrkräfte versuchen den Studenten Kenntnisse in diesem Bereich zu vermitteln, die einschlägigen Bücher aus der Bundesrepublik oder Österreich sind u. a. in den Lesesälen des Goethe-Instituts oder anderer ausländischer Kulturorganisationen zugänglich. Die Bildungslücke KJL füllt sich langsam auf, oft auch auf anderem Wege: Da die deutschsprachige KJL mittlerweile ein Teil der Popkultur geworden ist, können die jungen Rezipienten in Polen manche Werke dank ihren Verfilmungen (u. a. Johanna Spyris Heidi, Ellis Kauts Pumuckl oder die phantastischen Geschichten von Michael Ende, Cornelia Funke), aus Zeichentrickfilmen (z. B. Paul Maars Sams, Janoschs Paar der kleine Tiger und der kleine Bär) oder Theateradaptionen (z. B. Tankred Dorst und Ursula Ehlers Ameley, der Biber und der König auf dem Dach, Wie Dilldapp nach dem Riesen ging, König Sofus und das Wunderhubn) kennenlernen.

\section{Lehre}

In Polen sind sowohl Lehrangebote als auch germanistische Forschungen zur deutschsprachigen KJL selten. Vor 2008 versuchten Monika Hernik-Młodzianowska und Marta Ratajczak (2007: 157-176; 2008: 219-234) von der Germanistik in Zielona Góra einen Überblick über die Situation zu geben. Sie haben auf die wenigen polnischen Germanistinnen und Germanisten verwiesen, die sich mit verschiedenen Aspekten der KJL in ihren Dissertationen beschäftigt haben, sowie auf einige Initiativen, die KJL in den Lehrplänen und Bachelor- bzw. Magisterseminaren zu berücksichtigen (u. a. in Wrocław im Lehrstuhl für Didaktik des Deutschen und in Zielona Góra im Rahmen der Germanistischen Institutspartnerschaft mit der Universität Gießen und in Zusammenarbeit mit Prof. Carsten Gansel) (Hernik-Młodzianowska/Ratajczak 2008: 223-225). Aus ihren Beobachtungen ergibt sich folgendes Bild: Charakteristisch für die Vermittlung der KJL an polnische Germanistikstudenten sind ,Besprechungen 'von Texten, die Konzentration auf Inhalte und Problematik, eine nur beschränkte Auseinandersetzung mit narratologischen Problemen der Texte, mangelnde Eingliederung der Texte in die gesellschaftlichen Modernisierungsprozesse 
und die seltene Behandlung der im Zeitraum 1970-1995 entstandenen Werke. In die Untersuchungen wurden auch die damals noch existierenden dreijährigen Deutschkollegs wegen ihres Profils miteinbezogen. In der Lehrerausbildung in Opole wurde z. B. ein KJL-Programm angeboten, auf der Lektüreliste befand sich vorwiegend intentionale KJL, so mehrere Märchen verschiedener Autoren, einiges aus dem Bereich Volksliteratur und Sage, die als Sozialisationsmittel des 19. Jahrhunderts gedachten Texte von Heinrich Hoffmann und Wilhelm Busch, Texte von Erich Kästner, Bilderbücher von Janosch, einige bekannte Jugendromane der 1970er-1980er Jahre, ausgewählte Gedichte und Comics und TV-Produktionen für Kinder und Jugendliche. So wurde den künftigen Lehrerinnen und Lehrern eine Portion KJL-Wissen vermittelt, doch ohne die diesbezüglichen Entwicklungen der zwei letzten Jahrzehnte. Das Fach schien also der pädagogischen Ausbildung angepasst zu sein, wohl aber in der Überzeugung, die Absolventen würden es dann im Deutschsprachenunterricht nur sehr eingeschränkt anwenden können. Die Schulpraxis sieht nämlich für gewöhnlich so aus, dass wegen der bescheidenen Zahl der Wochenstunden als Literatur nur die im Lehrbuch stehenden, oft vereinfachten bzw. didaktisierten Auszüge bekannter Texte oder Gedichte behandelt werden, was andererseits auch mit dem Sprachniveau der Schüler zusammenhängt. Nur jene in zweisprachigen Klassen oder die Teilnehmer des als Deutscholympiade bekannten Sprachwettbewerbs wären im Stande, längere anspruchsvolle literarische Texte zu lesen.

Die in großen Städten befindlichen Bildungseinrichtungen können bei der Vermittlung und Popularisierung der deutschsprachigen KJL von der Zusammenarbeit polnischer Bibliotheken mit dem Goethe-Institut profitieren, zu dessen Rahmenprogramm auch Ausstellungen und Lesungen von Autorinnen und Autoren gehören - letztere sind besonders wichtig, da sie den Teilnehmern die Möglichkeit geben, den eingeladenen Gast zu seinen Texten zu befragen. Als ein Beispiel dafür können Lesungen mit Tamara Bach im Oktober 2015 und Lena Hach im Juni 2019 in Szczecin genannt werden, die anlässlich von Tagungen stattfanden, welche der Lehrstuhl für Deutschsprachige Gegenwartsliteratur der dortigen Universität und das Institut für Germanistik der Universität Gießen organisierten.

\section{Forschung}

Blickt man auf die in Polen im Rahmen der Germanistik tätigen Forscherinnen und Forscher, dürfen Publikationen, also Dissertationen und andere Monografien sowie Artikel in wissenschaftlichen Fachzeitschriften als Grundlage der Erörterung dienen.

Die von Hernik-Młodzianowska und Ratajczak (2008: 233) ermittelte Liste von Dissertationen (4) und wissenschaftlichen Projekten (2) war bescheiden. Sie umfasste lediglich die 1990er sowie die ersten Jahre nach 2000 und ist heute selbstverständlich nicht mehr aktuell. Danach sind einige weitere Monografien entstanden, u. a. die Dissertationen von Hernik-Młodzianowska (2012), Danuta Habdas (2014), Gerda Nogal (2014), Brygida Sobótka (2015), Małgorzata Kraśnik (2020) und die Habilitationsschrift von Małgorzata Filipowicz (2016). Es gibt keine Zweifel mehr, dass sich die Nachwuchswissenschaftler künftig noch der KJL-Problematik annehmen werden. 
Die Recherche in den Inhaltsverzeichnissen von germanistischen Zeitschriften ermöglicht eine differenziertere Betrachtung der Wahrnehmung deutschsprachiger KJL an polnischen Universitäten und Hochschulen. Die am längsten bestehenden Periodika begannen in den späten 1950er Jahren zu erscheinen (Germanica Wratislaviensia), in den nachfolgenden Jahrzehnten wurden weitere an fast jeder Hochschulgermanistik herausgegeben, heute sind es etwa 25 Titel. $^{3}$ Auch wenn hier nicht alle polnischen germanistischen Zeitschriften erfasst werden können, dürften die Ergebnisse der Recherche Auskunft darüber geben, inwieweit es bereits vor der Wende Interesse an der deutschsprachigen KJL im Lande gab, welche ihrer Themenbereiche einbezogen wurden und was sich diesbezüglich unter den neuen sozialen, wirtschaftlichen und politischen Umständen verändert hat.

Es fällt auf, dass vor 1989 Beiträge zur Kinder- und Jugendliteratur grundsätzlich eine Randerscheinung waren, solche Werke wurden bestenfalls erwähnt. Dies illustrieren folgende Beispiele: Hubert Orłowski spricht von der Jugendliteratur der NS-Zeit in einem Artikel (1973: 113) und Edyta Połczyńska (1979) behandelt die polnische Thematik in der jüngsten DDR-Literatur, darunter deutsch-polnische Liebesgeschichten, die auch von Jugendlichen gelesen werden. Für diese Zeit ist die bloße Erwähnung des Phänomens KJL charakteristisch, auf die Spezifik der Texte wird nicht eingegangen. Umfassendere Arbeiten sind ebenfalls eine Seltenheit, hier kann die Abhandlung von Norbert Honsza (1979) genannt werden, die u. a. Werke von Jules Verne, Science-Fiction und Comic-Literatur behandelt. Die spezifische KJL steht damals kaum im Fokus der germanistischen Forschung, die Aufmerksamkeit gilt vor allem älteren Werken, z. B. den Kinder- und Hausmärchen der Brüder Grimm und Karl May (Kunicki/Honsza 1986), oder Texten mit politisch aktuellem Hintergrund (Freundschaft VRP-DDR, deutsch-polnische Liebe). Es scheint, dass die nach $1970 \mathrm{im}$ Westen entstehende problemorientierte, realistische KJL in Polen noch unbekannt ist, was vielleicht mit dem damals erschwerten Zugang zu Neuerscheinungen zusammenhängt. Andererseits dürfte man auch die unter Neuphilologen nicht etablierte Tradition einer wissenschaftlichen Behandlung der KJL als Grund für die Abwesenheit einschlägiger Forschungen vermuten.

3 Für den Beitrag wurden folgende Periodika in Betracht gezogen: Germanica Wratislaviensia (1957-2019); Studia Germanica Posnaniensia (1971-2018); Lubelskie Materiaty Neofilologiczne (1974-2012); Acta Universitatis Nicolai Copernici. Nauki Humanistyczno-Spoteczne. Filologia germańska (1974-2006); Studia Niemcoznawcze / Studien zur Deutschkunde (1979-2018); Studia i materiaty. WSP Zielona Góra. Germanistyka (1986-1998); Colloquia Germanica Stetinensia (1988-2018); Rozprawy Niemcoznawcze. WSP Czestochowa (1991-1995); Studia Germanica Gedanensia (1993-2018); Zeszyty Naukowe Uniwersytetu Opolskiego. Filologia Germańska (1993-1996), Orbis Linguarum (1994-2018); Konfiguracje / Konfigurationen. Zeszyty Naukowe WSP w Bydgoszczy (1996-2003); Germanistyka. Uniwersytet Zielona Góra (2001-2004); Historia Literatury. Uniwersytet Rzeszowski (2002-2010), Historia Literatury. Tematy i Konteksty. Uniwersytet Rzeszowski (2011), Tematy i Konteksty. Uniwersytet Rzeszowski (2012-2017); Linguae Mundi. WSJO Poznań (2003-2014/2015); Litteraria Copernicana (2008-2019); Tekst i Dyskurs / Text und Diskurs. Uniwersytet Warszawski, Uniwersytet Rzeszowski (2008-2018); Thalloris. Philologische Studien / Philological Studies / Etudes philologiques. Uniwersytet Zielona Góra (2016-2017); Transfer. Reception Studies. Uniwersytet Częstochowa (2016-2018); Wortfolge / Szyk Stów. Uniwersytet Śląski (2017-2019); auch Serien: Posener Beiträge zur Germanistik, Danziger Beiträge zur Germanistik, Lodzer Arbeiten zur Literatur-und Kulturwissenschaft.

4 Orłowski 1973: 113: „Als ein minder bedeutsames Betätigungsfeld muss die Jugendliteratur gelten, die sich vor 1933 thematisch vor allem auf den Weltkrieg und auf,vaterländische Wendepunkte' konzentriert." 
Nach 1989 nimmt das Interesse der Germanistinnen und Germanisten an der KJL allmählich zu, doch nicht infolge veränderter Curricula, sondern privater Kontakte oder Lesepräferenzen, die auf den Gegenstand der wissenschaftlichen Arbeit übertragen werden. Impulse resultieren zum Teil aus der Expansion der KJL-Forschung in der Bundesrepublik, die Erträge der polnischen Germanistik auf diesem Gebiet sind jedoch noch bescheiden. Zu nennen sind u. a. Spezialistinnen und Spezialisten aus Łódź, Poznań, Szczecin, Warszawa, Wrocław und Zielona Góra. In fast allen Fachzeitschriften sind die Arbeiten breit fokussiert. Sie umfassen Gattungsfragen, Untersuchungen ausgewählter Themen- und Problemkomplexe, komparatistisch angelegte Untersuchungen, die Anwendung der KJL in der Didaktik oder Beziehungen zwischen dem literarischen Text und anderen Medien (Musik, Film, Internet), seltener theoretische Fragen, sprachwissenschaftlich profilierte Beiträge zur Lexik im Kinder- und Jugendbuch oder zu Übersetzungen deutscher Texte in andere Sprachen. Es werden bisherige Trends fortgesetzt, z. B. die Märchenforschung (u. a. in Publikationen von Katarzyna Grzywka), aber in den Beiträgen werden meistens die Gattung selbst, ihre Geschichte, Werke von Autoren der Romantik oder gegenwärtige Vertreter dieser literarischen Form behandelt, der Zusammenhang der Märchen mit kindlichen Rezipienten wird lediglich angestreift. Ähnlich ist es im Fall von Erich Kästner (anlässlich seines 100. Geburtstages: Hałub 1999) und Karl Mays Schaffen (es wird v. a. von Jacek Rzeszotnik nach thematischen Gesichtspunkten, als Abenteuer-, Unterhaltungs-, Trivialliteratur oder Bestsellerromane klassifiziert). Seit den 1990er Jahren hat die veränderte wirtschaftliche und politische Wirklichkeit großen Einfluss auf die Entscheidungen der Forscherinnen und Forscher, die nun die Möglichkeit haben, das aktuelle Angebot des westlichen Buchmarkts kennenzulernen, Forschungsstipendien zu beantragen, an themenrelevanten Konferenzen teilzunehmen, mit deutschen Universitäten und Kollegen zusammenzuarbeiten, dank den Internetausgaben renommierter Zeitungen und den dort gedruckten Rezensionen schnell die Neuerscheinungen wahrzunehmen, schließlich: die Bücher online zu bestellen.

Seit der Mitte der 1990er Jahre wird immer häufiger die spezifische KJL der zweiten Hälfte des 20. und des frühen 21. Jahrhunderts aufgegriffen, die historische und/oder gesellschaftliche Vorkommnisse verarbeitet. Es entstehen themenbezogene, autorenbezogene, literaturtheoretische, komparatistisch angelegte oder auch didaktisch orientierte Untersuchungen, die jene aus dem deutschsprachigen Raum ergänzen können. Manche Forscher spezialisieren sich auf eine bestimmte Problematik.

Die Germanistinnen und Germanisten von der Universität in Zielona Góra untersuchen speziell narratologische Aspekte der Kinder- und Jugendbücher, wofür die Partnerschaft mit der Justus-Liebig-Universität in Gießen 2005-2012 den Impuls gab, in deren Rahmen mehrere Tagungen zur KJL veranstaltet wurden (Gansel/Zimniak 2011a, 2011b). Sie konzentrieren sich u. a. auf die Thematik der Adoleszenz in der Literatur und Literaturdidaktik, das Schaffen von Peter Härtling, das Thema weibliche Adoleszenz, Neue-Pop-Literatur und die Erinnerungsproblematik in der KJL.

Die Germanistinnen von Szczecin beschäftigen z. B. folgende Fragen: die Gestaltung von deutsch-polnischen Wechselbeziehungen in der KJL, Migrationen, die neueste polnische KJL über Flüchtlinge, neue Medien und Technologie als Thema der KJL, All-AgeRomane. Am Lehrstuhl für Deutschsprachige Gegenwartsliteratur sind zwei Sammelbände 
in polnischer Sprache zur Popularisierung der deutschsprachigen KJL mit Beiträgen von Germanisten aus verschiedenen polnischen Hochschulen entstanden (Kamińska-Ossowska/ Hendryk 2016, 2017).

Die Sprachwissenschaftler, vor allem Sprachwissenschaftlerinnen, analysieren gattungstypische metaphorische Modelle im Kindergedicht sowie die Spezifik der Übersetzung (Chabros 2003; Urban 2012; Fimiak-Chwiłkowska 2012).

Im Allgemeinen lässt sich eine wachsende Beliebtheit der sog. Neuen Pop-Literatur bei polnischen Germanistinnen und Germanisten beobachten, es entstehen Artikel über Romane von Christian Kracht, Marc Fischer, Sibylle Berg, Alexa Hennig von Lange, Malin Schwerdtfeger, Silke Scheuermann, Zoë Jenny, Katja Oskamp.

Im Rahmen der gattungsbezogenen Arbeiten ist das Interesse an Bilderbuch (u. a. in Bezug auf Illustrationen) (Teodorowicz-Hellman 2003; Sommerfeld 2018) und Comic sichtbar. Während bei der ersten Form die Assoziation mit der KJL offensichtlich ist, ist es in den letzten Jahrzehnten zu einer derartigen Ausdifferenzierung der Comics und Graphic Novels gekommen, dass sie eher an ältere Jugendliche und junge Erwachsene gerichtet sind und somit nicht (mehr) als ein Bestandteil der KJL betrachtet werden (dürfen).

Es fällt auf, dass die Forscherinnen und Forscher oft ausgewählte Probleme, die Erzähltechnik oder Form- und Gattungsspezifik der (auch an Kinder oder Jugendliche gerichteten) Texte untersuchen und dabei die Jugendliteratur als solche nicht separat betrachten. ${ }^{5}$ An vielen Titeln von wissenschaftlichen Publikationen lässt sich nicht erkennen, dass sie auch der breit verstandenen KJL gelten.

Zusammenfassend können im Rahmen der polnischen germanistischen Forschung zur KJL im Allgemeinen folgende Positionen unterschieden werden:

- Publikationen zur spezifischen KJL, etwa Beiträge in Fachzeitschriften oder Monografien zu einem Autor oder einem Problem bei mehreren Autoren;

- monografische Publikationen zu einem Autor, in dessen Werk sich Kinder- und Jugendbücher befinden, die aber bestenfalls am Rande als solche behandelt werden;

- Untersuchungen zur Abenteuer-, Unterhaltungs- oder Trivialliteratur, die auch die Jugendliteratur umfassen, aber deren Spezifik oder gar den Begriff außer Acht lassen;

- Untersuchungen zur Form oder Erzählweise der Texte, in denen deren Zugehörigkeit zur KJL relativ selten im Vordergrund steht, häufig nur erwähnt wird;

- komparatistische Untersuchungen zur deutschen und polnischen KJL;

- Untersuchungen zu Illustrationen im Kinderbuch;

- selten: sprachwissenschaftliche Untersuchungen zu einem KJL-Text;

- Arbeiten zur Anwendung der KJL im Lehrprozess.

\footnotetext{
5 Informationen über Bücher für junge Leser werden oft in Abhandlungen zu Autoren und Themen ergänzend eingeflochten. Z. B. stehen bei der Behandlung der Werke von Leonie Ossowski (Orłowski 1995: 5: Bezug auf Die große Flatter und Stern ohne Himmel) und von Marlen Haushofer (Palmer 1993) Hinweise auf Texte für Kinder und Jugendliche, aber deren Problematik wird nur kurz kommentiert und nicht als KJL-typisch untersucht. Manche von Palmer als Frauenromane eingestuften Texte, so Himmel, der nirgendwo endet, Eine Handvoll Leben, könnte man auch der Literatur für junge Erwachsene bzw. der All-Age-Literatur zuordnen. Ähnliches gilt auch für Bezüge auf Jugendromane über Flüchtlinge (Wolting 2019).
} 
Die vor einigen Jahrzehnten als nachrangig empfundene KJL ist heute zum unbestrittenen Bestandteil der Forschung polnischer Germanistinnen und Germanisten geworden, auch wenn in vielen Publikationen die Auseinandersetzung mit ihr unter anderen Prämissen erfolgt und die Zuordnung zu diesem Subsystem vermieden wird. Wird sie als Lehrgegenstand noch etwas vernachlässigt, ist sie doch kein Untersuchungsgegenstand zweiten Ranges mehr und findet Interesse im Kontext der allgemeinen literaturwissenschaftlichen wie auch der interdisziplinären Forschung.

\section{Schlussbemerkungen}

Die deutschsprachige Kinder- und Jugendliteratur ist in Polen keine unbekannte Erscheinung mehr. Die Öffnung des Buchmarktes in den 1990er Jahren hat neue Möglichkeiten geschaffen: die Selbstbestimmung der Verlage, eine Förderung der Übersetzer, internationale Workshops, Kontakte der Übersetzer mit Autoren. Die Erweiterung der älteren Definition der KJL um neue Komponenten, vor allem die Verschiebung der Grenze des Jugendalters in die Erwachsenenwelt, bedeutet ein größeres Publikum für die Texte und mehrere literarische Gattungen im Angebot. Die gut bekannten, älteren Werke begleiten gegenwärtige Texte, die Trivialliteratur ist zu einem festen Bestandteil der KJL-Regale in Buchhandlungen und Bibliotheken geworden. Die Verlage reagieren schnell auf Entwicklungen des westlichen Buchmarktes, Listen preisgekrönter Texte werden ja regelmäßig publiziert und können im Ausland als Impuls zur Übersetzung dienen. Bekannte Autoren werden oft zu Medienstars, viele suchen direkten Kontakt mit ihren Lesern, ihre Webseiten sind überall zugänglich.

Die Beschäftigung der polnischen Germanistinnen und Germanisten mit allen Phänomenen der KJL hat sich ab den 1990er Jahren merklich intensiviert. Sie profitieren viel vom Gedankenaustausch und von Kontakten mit deutschen Kollegen, auch wenn ein breiterer organisatorischer bzw. konzeptueller Rahmen nicht vorhanden ist. Es bleibt auf die weitere Entwicklung der polnischen KJL-Forschung im Rahmen des internationalen wissenschaftlichen Diskurses zu hoffen.

\section{Literatur}

Chabros, Eliza (2003): Analyse gattungstypischer metaphorischer Modelle im neueren deutschsprachigen Kindergedicht. In: Lubelskie Materiaty Neofilologficzne. 27, 61-71.

Degler, Frank / Paulokat, Ute (2008): Neue Deutsche Popliteratur. Paderborn: Wilhelm Fink.

Doderer, Klaus (1981): Kinder- und Jugendliteratur im Ghetto? In: Klaus Doderer (Hg.): Ästhetik der Kinderliteratur. Plädoyers für ein poetisches Bewußtsein. Mit Beiträgen von Dieter Arendt, Klaus Doderer, Klaus F. Geiger, Dietrich Grünewald, Bettina Hurrelmann, Winfred Kaminski, Wolfgang Promies, Joseph H. Schwarcz, Gert Ueding und Reiner Wild. Weinheim, Basel: Beltz Verlag, 9-17.

Doderer, Klaus (1992): Literarische Jugendkultur. Kulturelle und gesellschaftliche Aspekte der Kinderund Jugendliteratur in Deutschland. Weinheim, München: Juventa Verlag. 
Ewers, Hans-Heino (1995): Die Emanzipation der Kinderliteratur. Anmerkungen zum kinderliterarischen Funktionswandel seit Ende der 60er Jahre. In: Renate Raecke, Ute D. Baumann (Hg.): Zwischen Bullerbü und Schewenborn. Auf Spurensuche in 40 Jahren deutschsprachiger Kinder-und Jugendliteratur. München: Arbeitskreis für Jugendliteratur e.V., 16-28.

Filipowicz, Małgorzata (2016): Lustige Gespenster. Komik im Kinder-und Jugendbuch von Christine Nöstlinger. Warszawa: Instytut Germanistyki Uniwersytetu Warszawskiego.

Fimiak-Chwiłkowska, Anna (2012): Die Welt in Kinderworten. Zum Problem der Übersetzung der Kinderliteratur am Beispiel des Romans „Król Maciuś I“ (1923) von Janusz Korczak in zwei deutschen Übersetzungen. In: Studia Germanica Gedanensia. 27, 196-210.

Gansel, Carsten (1995): Zum kulturellen Wandel kindlicher und jugendlicher Lebenswelten und ihrer Reflexion in der Kinder- und Jugendliteratur. In: Mitteilungen des deutschen Germanistenverbandes. $42(3), 11-18$.

Gansel, Carsten (2016): Moderne Kinder-und Jugendliteratur. Vorschläge für einen kompetenzorientierten Unterricht (7. Aufl.). Berlin: Cornelsen.

Gansel, Carsten / Zimniak, Paweł (Hg.) (2011a): Zwischen didaktischem Auftrag und grenzüberschreitender Aufstörung? Zu aktuellen Entwicklungen in der deutschsprachigen Kinder-und Jugendliteratur. Heidelberg: Universitätsverlag Winter.

Gansel, Carsten / Zimniak, Paweł (Hg.) (2011b): Zwischenzeit, Grenzüberschreitung, Aufstörung. Bilder von Adoleszenz in der deutschsprachigen Literatur. Heidelberg: Universitätsverlag Winter.

Habdas, Danuta (2014): Die Rezeption der literarischen Werke von Janosch (Horst Eckert) in Deutschland und Polen im Kontext der autobiografischen und regionalen Aspekte seines Schaffens Poznań: Uniwersytet im. Adama Mickiewicza (unveröffentlichtes Manuskript).

Hałub, Marek (1999): Erich Kästner. W 100 rocznicę urodzin [Erich Kästner. Zum 100. Geburtstag]. In: Orbis Linguarum. 12, 45-51.

Hernik-Młodzianowska, Monika (2012): Zur Inszenierung von Erinnerung im Werk von Peter Härtling. Zielona Góra: Oficyna Wydawnicza Uniwersytetu Zielonogórskiego.

Hernik-Młodzianowska, Monika / Ratajczak, Marta (2007): Kinder- und Jugendliteratur in Curricula an polnischen Universitäten. In: Edward Białek, Czesław Karolak (Hg.): Schubnummer oder Leben! Beiträge zur Literaturkritik und zum kinder-und jugendliterarischen Schriftum. [Orbis linguarum. Beihefte zu Orbis linguarum: 60]. Dresden, Wrocław: Neisse Verlag, 157-176.

Hernik-Młodzianowska, Monika / Ratajczak, Marta (2008): Kinder- und Jugendliteratur in Forschung und Lehre an polnischen Universitäten. In: Carsten Gansel, Paweł Zimniak, Karl W. Bauer (Hg.): Der Bologna-Prozess. Konsequenzen für die germanistische Ausbildung im internationalen Rabmen. Hohengehren: Schneider, 219-234.

Hohmeister, Elisabeth (1995): Vom kleinen Liebling zur Rotznase. Der ästhetische Wandel in der Darstellung von Kindern. In: Renate Raecke, Ute D. Baumann (Hg.): Zwischen Bullerbü und Schewenborn. Auf Spurensuche in 40 Jahren deutschsprachiger Kinder-und Jugendliteratur. München: Arbeitskreis für Jugendliteratur e.V., 61-69.

Honsza, Norbert (1979): Moderne Unterhaltungsliteratur. Bestandsaufnahme - Thesen - Analysen. In: Germanica Wratislaviensia. XXXVIII.

Kamińska-Ossowska, Ewelina / Hendryk, Ewa (Hg.) (2016, 2017): Oswoić rzeczywistość. Wokót niemieckojęzycznej literatury dla dzieci i mtodzieży [Mit der Wirklichkeit vertraut werden. Über die deutschsprachige Kinder-und Jugendliteratur]. Bd. I, Szczecin: volumina.pl; Bd. II, Szczecin: volumina.pl. 
Kraśnik, Małgorzata (2020): Zum Motiv des Todes in der deutschsprachigen Kinder-und Jugendliteratur (KJL) der Gegenwart. Zu ibrer Rolle und Funktion im Kontext der Erweiterung des Kulturwissens und der Perfektionierung der Sprachfertigkeiten der Schüler. Poznań: Instytut Filologii Germańskiej (Dissertation, unveröffentlichtes Manuskript).

Kunicki, Wojciech / Honsza, Norbert (1986): Karol May - Anatomia sukcesu. Życie, twórczość, recepcja [Karl May - Anatomie des Erfolgs. Leben, Werk, Rezeption]. Katowice: Wydawnictwo Śląsk.

Lange, Günter (2002a): Taschenbuch der Kinder-und Jugendliteratur. Bd. I: Grundlagen - Gattungen (3. unveränderte Aufl.). Baltmannsweiler: Schneider-Verl. Hohengehren, 158-565.

Lange, Günter (2002b): Taschenbuch der Kinder-und Jugendliteratur. Bd. II: Medien und Sachbuch, ausgewäblte thematische Aspekte, ausgewähltepoetologische Aspekte, Produktion und Rezeption, KJL im Unterricht (3. unveränderte Aufl.). Baltmannsweiler: Schneider-Verl. Hohengehren, 586-656.

Nogal, Gerda (2014): Weibliche Adoleszenz in der neuesten deutschsprachigen Literatur. Narratologische Fallstudien. Dresden, Wrocław: Neisse.

Orłowski, Hubert (1973): Die Herausbildung der faschistischen Literatur in den Jahren 1925-1933. In: Studia Germanica Posnaniensia. 2, 99-118.

Orłowski, Hubert (1995): Grenzen benennen - Grenzen überschreiten. Zum Werk von Leonie Ossowski. In: Orbis Linguarum. 3, 5-13.

Palmer, Katarzyna (1993): Das Problem der Einsamkeit in den Romanen von Marlen Haushofer. In: Studia Germanica Posnaniensia. 19, 15-22.

Połczyńska, Edyta (1979): Polnische Thematik in der jüngsten DDR-Literatur. In: Studia Germanica Posnaniensia. 8, 3-12.

Schönfeldt, Sybil Gräfin (1995): Autoritär - antiautoritär - und dann? Erziehungsmuster in Kinderbüchern. In: Renate Raecke, Ute D. Baumann, (Hrsg.): Zwischen Bullerbü und Schewenborn. Auf Spurensuche in 40 Jahren deutschsprachiger Kinder-und Jugendliteratur. München: Arbeitskreis für Jugendliteratur e.V., 119-126.

Sobótka, Brygida (2015): „Spogladatam w otchtan życia. To, co tam ujrzatam, nie byto piękne”. Alexy Hennig von Lange dialog z rzeczywistościa [„Ich blickte in den Abgrund des Lebens. Was ich dort sah, war nicht schön." Alexa Hennig von Langes Dialog mit der Wirklichkeit]. Warszawa: Instytut Germanistyki Uniwersytetu Warszawskiego.

Sommerfeld, Beate (2018): Obcość oswojona - transfer ilustrowanych książek dla dzieci między kulturą polską a niemiecką [Vertraute Fremde. Bilderbuchtransfer zwischen der polnischen und der deutschen Kultur]. In: Orbis Linguarum. 48, 319-329.

Stanz, Jörg / Weinmann, Andrea (2002): Die Kinder- und Jugendliteratur der Bundesrepublik nach 1945. In: Günter Lange (Hg.): Taschenbuch der Kinder-und Jugendliteratur. Bd. I: Grundlagen Gattungen (3. unveränderte Auf.). Baltmannsweiler: Schneider-Verl. Hohengehren, 97-136.

Teodorowicz-Hellman, Ewa (2003): Die Rolle der Illustrationen bei der Interpretation übersetzter Kinderund Jugendliteratur. Am Beispiel der polnischen Übersetzungen von Selma Lagerlöfs, „Wunderbare Reise des kleinen Nils Holgersson mit den Wildgänsen“. In: Studia Germanica Posnaniensia. 29, 177-191.

Urban, Anna (2012): Eigennamen und Titel in der Übersetzung der Kinder- und Jugendliteratur vor dem Hintergrund ihrer lokalisierenden Funktion. In: Studia Germanica Gedanensia. 27, 184-195.

Wolting, Monika (2019): Der neue Kriegsroman. Repräsentationen des Afghanistankrieges in der deutschen Gegenwartsliteratur. Heidelberg: Universitätsverlag Winter. 